\title{
Intermittent statistics and stochastic modelling of low and high Re compressible jets
}

\author{
Roberto Camussi *, Gaetano Luca Micci ${ }^{\dagger}$, Stefano Meloni ${ }^{\ddagger}$ \\ University of RomaTre, Department of Engineering, via della Vasca Navale 79, 00146, Rome, Italy \\ Christophe Bogey ${ }^{\S}$ \\ Univ Lyon, Ecole Centrale de Lyon,INSA Lyon, Université Claude Bernard Lyon I, CNRS, Laboratoire de Mécanique \\ des Fluides et d'Acoustique, UMR 5509, F-69134, Ecully, France
}

\begin{abstract}
In this work, we studied the intermittent statistics of pressure fluctuations in the near field of compressible subsonic single stream jets. The analysis has been performed considering data from numerical simulations of two isothermal jets having the same Mach number, $M=0.9$, and two different Reynolds number $(3,125$ and 100,000). Pressure fluctuations were measured by virtual probes located in different positions in the radial and axial directions. Intermittent events are extracted through appropriate wavelet-based indicators and two random variables are analyzed, namely the intermittent time $\Delta t$, that is the time delay between two successive events, and the energy amplitude $A$. Their statistics are computed with the scope of adressing the stochastic model proposed by Camussi et al. [1] that was settled up on the basis of experimental data. The present analysis extends the validity of the model to very low Reynolds number conditions and to the statistics of the $0^{\text {th }}$ azimuthal mode of the pressure fluctuations that exhibit a relevant degree of intermittency around the Kelvin-Helmholtz frequency.
\end{abstract}

\section{Introduction}

Subsonic jet noise has been a hot topic in the last 50 years because of its relevant implications in the design of modern civil aircraft tackling the problem of minimizing the noise impact. Since the publication of the Lighthill's famous paper [2], many researchers investigated jet induced pressure fluctuations both in the near-field and in the far-field in the attempt of developing models able to predict as accurately as possible the emitted noise. This large body of the literature clarified the nature of pressure events that dominate both fields. The far-field in the subsonic case is acoustic, with a Gaussian distribution of pressure fluctuations whereas hydrodynamic fluctuations induced by vortex structures generated by the interaction of the jet flow with the ambient fluid at rest dominate the near-field [3]. According to the typical statistics of vorticity in turbulence, the near-field pressure fluctuations exhibit a non Gaussian intermittent behaviour. In a statistical sense, intermittency is intended as a sequence of quiescent phases interrupted by active events inducing a non-homogeneous distribution of energy in time. This is recognized as a very efficient mechanism in generating acoustic waves and thus in contributing to jet noise. Indeed, the importance of intermittency in the noise generation mechanism of subsonic jets has been recognized since the seminal experiment undertaken by Juvé et al. [4] and confirmed in several successive studies (e.g. [5-10]). They demonstrated the existence and the dominant role played by intermittent events on the noise emitted by a jet. Recent papers (see among many, [11-13]) also highlighted the relevant contribution of turbulence-induced intermittency and the impact of non-linearities to the generation of noise.

The results reported by Kearney-Fischer et al. [14, 15] further support the idea that intermittent events are the dominant feature of jet noise. They applied a method to extract the events and developed stochastic models to reproduce their statistics in both the physical and the Fourier domain. A similar approach was adopted by Camussi et al. [1, 16] who used wavelet transform to select intermittent events from experimental data and proposed stochastic models to reproduce their relevant statistics. These analyses provided a direct measure of the degree of intermittency contained in the pressure field induced by compressible jets.

The main objective of the present work is to assess and validate the model proposed in [1, 16] by processing a pressure data-base obtained numerically. With respect to the recent paper by [17], where the same data-base was

\footnotetext{
*Roberto Camussi, Full professor, Department of engineering, University of Roma Tre, roberto.camussi@uniroma3.it

$\dagger \mathrm{PhD}$ student, Department of engineering, University of Roma Tre, gaetano.micci@uniroma3.it

*Research Fellow, Department of Engineering, University of Roma Tre, stefano.meloni@uniroma3.it

${ }^{\S}$ CNRS Research Scientist, Ecole Centrale de Lyon, INSA Lyon, Université Claude Bernard Lyon I, CNRS, Laboratoire de Mécanique des Fluides et d'Acoustique, christophe.bogey@ec-lyon.fr.
} 
analyzed, the present study uses exactly the same approach proposed in [1, 16] so that the procedure and the proposed model is definitely validated and extended to flow conditions that were never tested before, including the $0^{\text {th }}$ mode component extracted through a Fourier azimuthal decomposition of the pressure signals.

The paper is organized as follows: section II reports the numerical setup and the post-processing procedure, results are discussed in section III, whilst final remarks are reported in section IV.

\section{Numerical setup and post-processing procedure}

\section{A. Numerical setup}

The data-base analyzed has been obtained numerically through DNS and LES of two single-stream circular jets, having respectively diameter-based Reynolds numbers of 3,125 and 100,000 and Mach number 0.9 . Both jets originate from a pipe nozzle of length $\mathrm{D}$ into a medium at ambient temperature and pressure $293 \mathrm{~K}$ and $10^{5} \mathrm{~Pa}$. The potential core length is equal to 7.3D for both jets (see [18] for the details). The simulation times after the transient periods are equal to $0,500 \mathrm{D} / \mathrm{U}$ for the high $\mathrm{Re}$ jet and to $1,000 \mathrm{D} / \mathrm{U}$ for the low Re jet. Pressure time series are obtained for the azimuthal angles that varies from 0 up to $315^{\circ}$ by step of $45^{\circ}$ for the high Re jet and from from 0 up to $270^{\circ}$ by step of $90^{\circ}$ for the low Re jet. Signals are recorded at a sampling frequency allowing spectra to be computed up to $S t_{D}=6.4$. More details about the numerical simulations are reported in [18-20]. Pressure and velocity fluctuations are extracted from virtual probes positioned in the near field of the jets (of diameter D) displaced along the longitudinal (x) and radial (r) directions.

The following table reports the location of the virtual probes considered for the presented analysis:

\begin{tabular}{cccc}
\hline $\mathrm{M}$ & $\mathrm{Re}$ & $\mathrm{x} / \mathrm{D}$ & $\mathrm{r} / \mathrm{D}$ \\
\hline 0.9 & 100.00 & from 1 up to 7 by step of 1 & from 0.5 up to 1.5 by step of 0.5 \\
0.9 & 3,125 & from 1 up to 7 by step of 1 & from 0.5 up to 1.5 by step of 0.5 \\
\hline
\end{tabular}

It can be observed that the study is limited to a domain close to the jet exit, usually recognized as the noise-producing region of the jet flow and thus of relevant interest for jet-noise modelling. To this purpose, the original pressure signals are also represented in terms of their azimuthal components through the azimuthal decomposition of the Fourier modes. This approach has been extensively used in the past to extract relevant features connected with the jet noise generation [21]. The wavelet-based statistical analysis is thus applied also to the axisymmetric mode of order 0 , that is known to dominate the sound field for low polar angles [22].

\section{B. Post-processing procedure}

As performed in [1], signals are analyzed using a wavelet-based approach. The wavelet decomposition allows for the simultaneous representation of a temporal signal in terms of a time shift $(\mathrm{t})$ and a resolution time scale (s) whose inverse corresponds to the frequency (f). Formally, the wavelet transform of the signal $\mathrm{p}(\mathrm{t})$ is given by the following expression [23]:

$$
w(s, t)=C_{\psi}^{-\frac{1}{2}} s^{-\frac{1}{2}} \int_{-\infty}^{\infty} p(\tau) \psi^{*} \psi^{*}\left(\frac{t-\tau}{s}\right),
$$

where $s$ is the wavelet scale, $\tau$ is a time shift, $C_{\psi}^{-\frac{1}{2}}$ is a constant that takes into account the mean value of $\psi(t)$ and $\psi^{*}\left(\frac{t-\tau}{s}\right)$ is the complex conjugate of the dilated and translated mother wavelet $\psi(t)$. In this analysis, we applied the continuous wavelet transform (CWT) using the Morlet mother wavelet.

A relevant outcome of the wavelet trasform is the wavelet scalogram, given by the square of the wavelet coefficients.An example is reported in figure 1 (a) obtained by analyzing the pressure data taken at $x / D=2$ and $r / D=1$. It provides a decomposition of the energy onto the (f,t) plane, thus allowing us to determine the time evolution of energetic features contained into the signals (see e.g. [24]). Indeed, it should be pointed out that the Fourier power spectral density of the signal can be retrieved by integrating the scalogram into the time domain and an example of a comparison between the Fourier and the wavelet-reconstructed spectrum is provided in figure 1 (b) showing a striking agreement.

In jets, in the region close to the nozzle exit, a spectral bump can be clearly identified especially, for laminar exit conditions. It is the trace of the Kelvin-Helmholtz instability mode and the corresponding frequency and spectral amplitude are denoted as $f_{K H}$ and $A_{K H}$ respectively. 

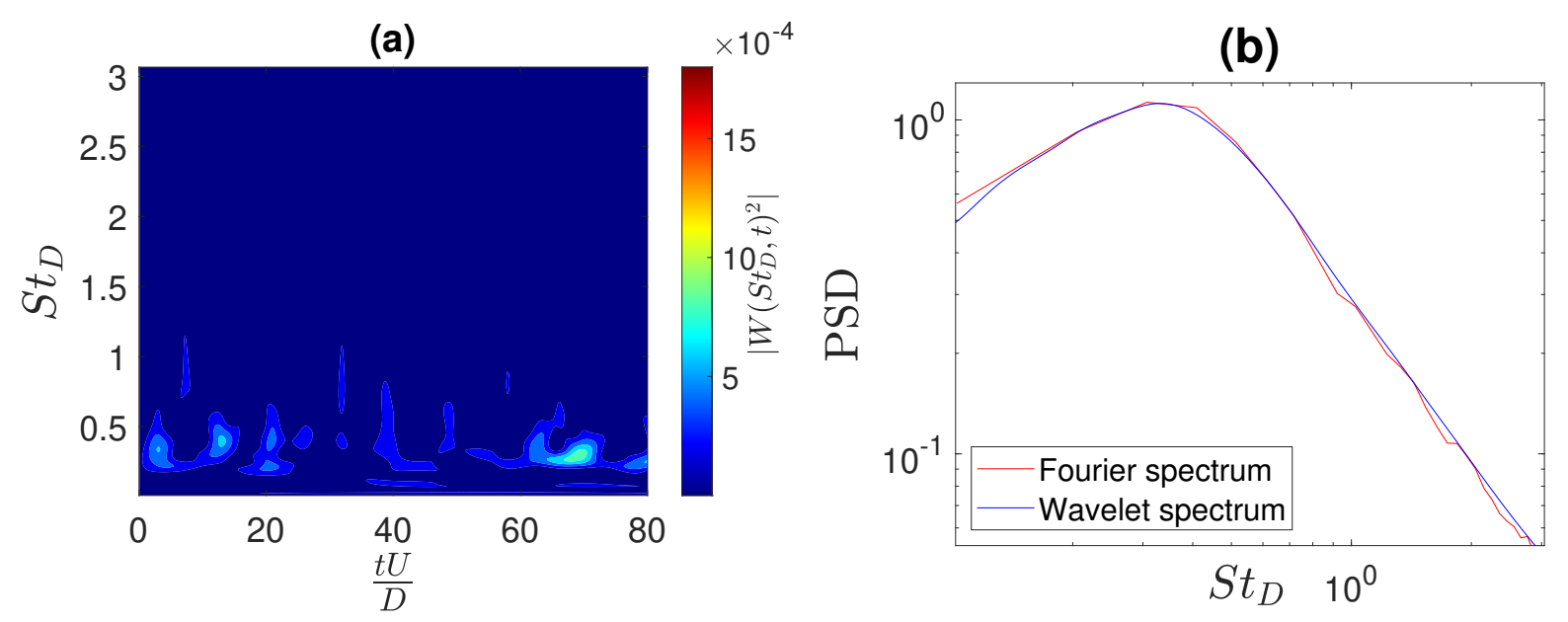

Fig. 1 (a) Wavelet scalogram at $x / D=2$ and $r / D=1$; (b) comparison between the Fourier spectrum and the wavelet-reconstructed spectrum obtained by integrating the case (a).

The quantity used to identify intermittent events is the so-called Local Intermittency Measure (LIM) that represents a normalized version of the wavelet scalogram. Its formal definition is the following:

$$
\operatorname{LIM}(s, t)=\frac{w^{2}(s, t)}{<w^{2}(s, t)>_{t}},
$$

where $w^{2}(s, t)$ are the wavelet coefficients evaluated with eq. 1) whilst the symbol $<\ldots>_{t}$ indicates time average. According to [1, 16], the LIM is computed at the wavelet scale corresponding to $f_{K H}$ and the condition $L I M>1$ is used to identify intermittent events having a local energy greater than the average. We indicate with $\Delta_{t}$ the time between two consecutive intermittent events, defined as intermittent time, and with A the event energy amplitude retrieved from the square of the corresponding wavelet coefficient. These the two indicators will be characterized in details in the following.

\section{Results}

The number of intermittent events depends on the flow conditions considered and on the position of the selected virtual probes. As shown in figure 2, in the region close to the jet exit, the number of intermittent events decreases for increasing both the axial and the radial distances whereas it is larger for the lower Reynolds number. This is in agreement with results by Bogey et al. [17] that highlighted a large degree of intermittency at low $R e$.

A similar trend is observed for the statistics of the 0 mode, as reported in Figure 3 . Even though the relative numbers of selected events is lower with respect to the full pressure, the 0 mode intermittency also shows a decreasing trend for increasing both $x / D$ and $r / D$ whereas it is larger at the lowest $R e$.

Following the previous work of Camussi et al. [16], averaged values of both the intermittent time and the amplitude are computed and are used as reference for a simplified model.

We report in figure 4 (a) the ratio $<A>/ A_{K H}$ against x/D for the low $R e$ case, where $<A>$ is the mean amplitude of the detected intermittent events and $A_{K H}$ is the amplitude of the corresponding Kelvin-Helmholtz (K-H) mode. As a first approximation, a reference constant value can be assumed by averaging the whole set of available data (the continuous line in figure 4).

The analogous results in terms of $S t_{\text {int }}$ as reported in figure 4(b). We remind that, according to [16], the quantity $S t_{\text {int }}$ is defined using as a reference time-scale the mean intermittent time $\Delta t$, and $S t_{K H}$ is the mean intermittent non-dimensional time computed at the $\mathrm{K}-\mathrm{H}$ frequency.

The averaged values of the normalized intermittent time $S t_{i n t} / S t_{K H}$ and amplitude $A / A_{K H}$, including the higher $R e$ cases, are summarized in table 1 showing a weak dependence upon $R e$.

Similar results are obtained in the case of the $0^{t h}$ mode. The normalized amplitudes and Strouhal numbers are reported in Figure 5 and the mean values are summarized in table 2 showing a very good agreement with results obtained from the full pressure signals. 
(a)

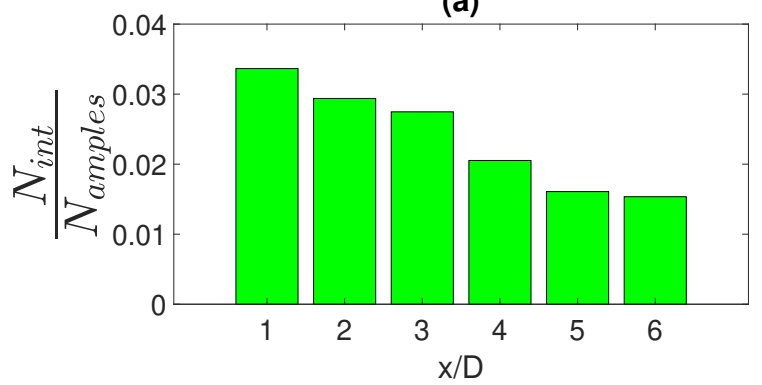

(b)

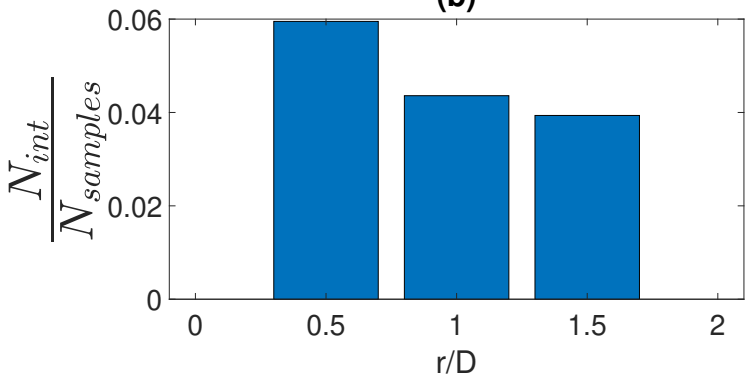

(c)

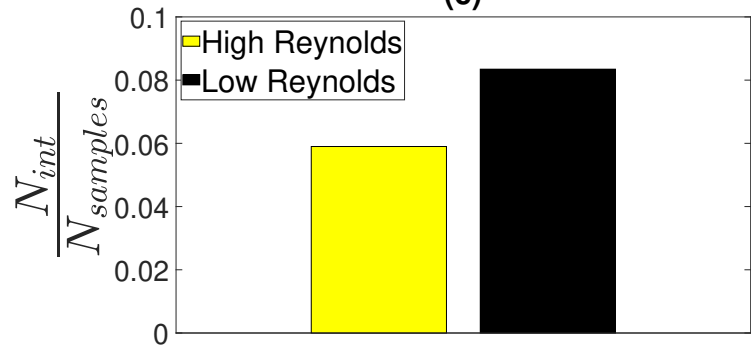

Fig. 2 Number of intermittent events normalized with respect to the number of samples: (a) as a function of $\mathrm{x} / \mathrm{D}$ for all $\mathrm{r} / \mathrm{D}$ and both Reynolds numbers; (b) as a function of $\mathrm{r} / \mathrm{D}$ for all $\mathrm{x} / \mathrm{D}$ and both Reynolds numbers; (c) as a function of Reynolds numbers for all radial and axial positions.

(a)

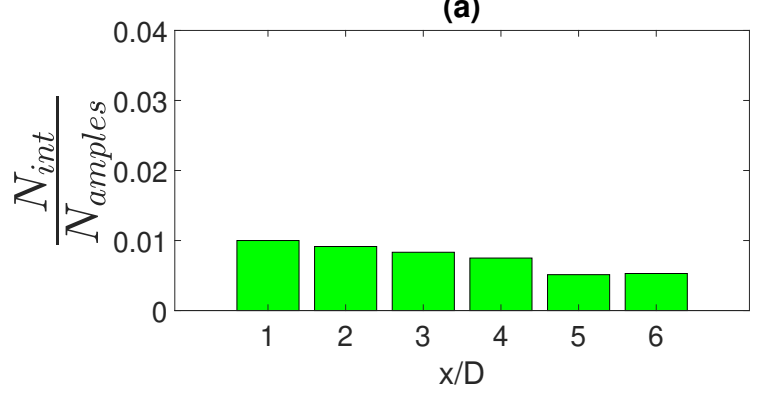

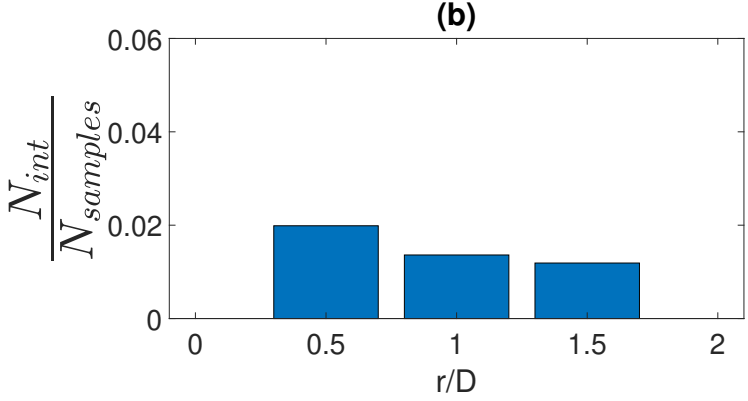

(c)

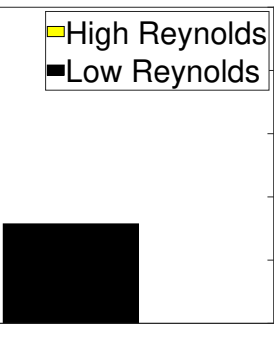

Fig. 3 Same as Figure 2 but for the 0 mode. 
(a)

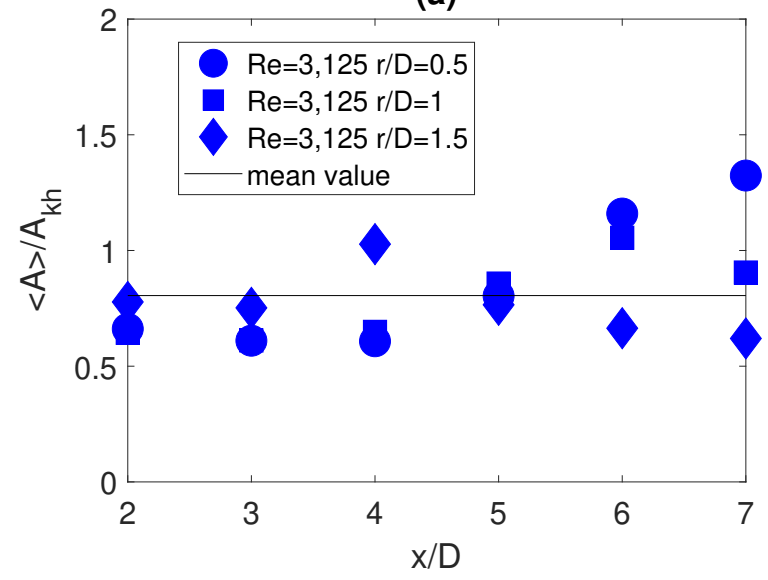

(b)

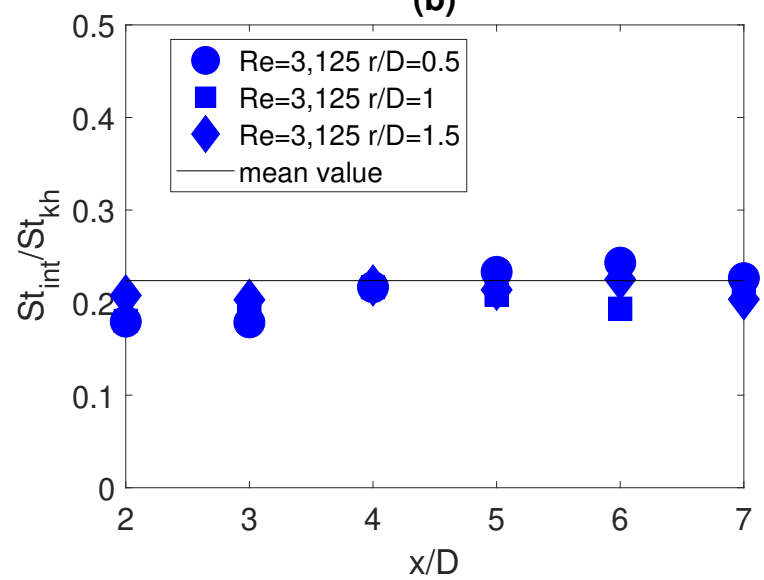

Fig. 4 Axial evolution of (a) $A_{i n t} / A_{K H}$ and (b) $S_{i n t} / S_{K H}$ at the lowest $R e$ and different $r / D$. 
(a)

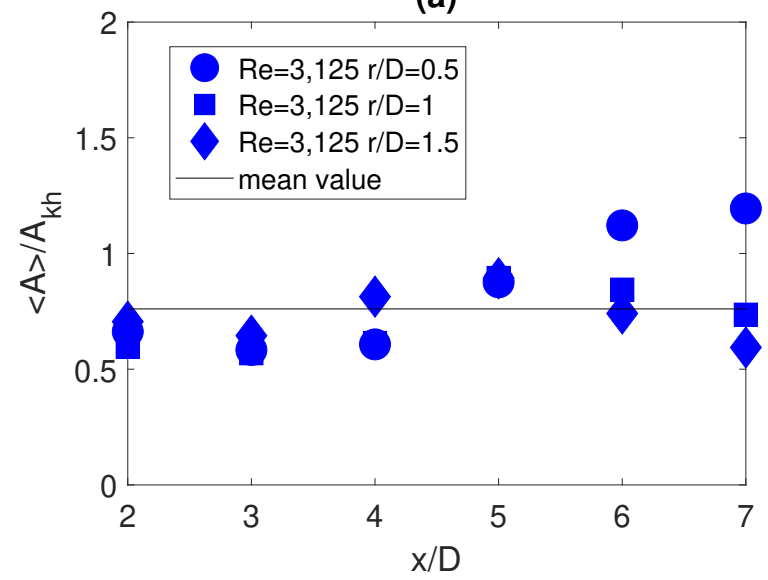

(b)

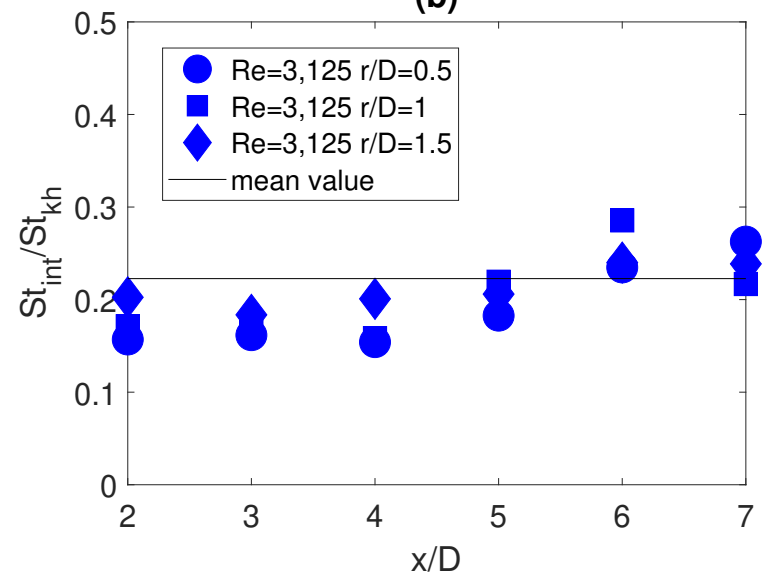

Fig. 5 Same as Figure 4 but for the mode 0. 
This result provides a first indication that a stochastic model based on the full pressure signals can be extended to the $0^{\text {th }}$ mode intermittent statistic, a relevant feature in view of the direct correlation between the $0^{\text {th }}$ mode properties and the noise generation mechanisms.

\begin{tabular}{cc}
\hline Pressure & Mean \\
\hline$S t_{i n t} / S t_{K H}$ & 0.223 \\
High Reynolds $<A>/ A_{K H}$ & 1.00 \\
Low Reynolds $<A>/ A_{K H}$ & 0.80 \\
\hline
\end{tabular}

Table 1 Statistics of the intermittent events identified in the original pressure signals.

\begin{tabular}{cc}
\hline Pressure $0^{\circ}$ azimuthal mode & Mean \\
\hline$S t_{\text {int }} / S t_{K H}$ & 0.227 \\
High Reynolds $<A>/ A_{K H}$ & 0.91 \\
Low Reynolds $<A>/ A_{K H}$ & 0.76 \\
\hline
\end{tabular}

Table 2 Statistics of the intermittent events identified in the $0^{\text {th }}$ mode pressure signals.

The detailed statistical distribution of the intermittent events is determined by calculating the Probability Density Functions (PDF) of $\Delta t$ and A. The main purpose of this analysis is to evaluate the dependence of these PDFs, both for the whole pressure signals and for the $0^{\text {th }}$ mode, upon the considered flow parameters (Reynolds number, axial position, radial distance).

The PDFs of the numerical data are approximated using the same analytical model proposed in [16]. More specifically, the analytical forms are provided in terms of the normalized reduced variables $\Delta t^{*}$ and $A^{*}$ that are defined as follows:

$$
\begin{gathered}
\Delta t^{*}=\frac{\Delta t-<\Delta t>}{\sigma_{\Delta t}} \\
A^{*}=\frac{A-<A>}{\sigma_{A}}
\end{gathered}
$$

and the functions proposed in [16] (see equations 5] and 6 consist of an hyperbolic secant to approximate the PDF of $\Delta_{t}$ and a decaying exponential function for the PDFs of $A$ :

$$
\begin{gathered}
P D F\left(\Delta t^{*}\right)=a_{1} \operatorname{sech}\left(b_{1} \Delta t^{*}\right), \\
P D F\left(A^{*}\right)=a_{2} e^{-b_{2} A^{*}},
\end{gathered}
$$

Figure 6 (a) and (b) reports the PDFs of the variables $\Delta t$ and $A$ respectively, obtained from the whole set of virtual probes analyzed therein. The shape of the PDFs do not change significantly and the analytical approximations (5) and (6) are reported in Figure 6 (c) and (d) along with an uncertainty interval estimated from the experimental data dispersion. The amplitude of the uncertainty is not relevant thus confirming the reliability of the model. The coefficients of the approximating functions are:

$a_{1}=0.56, b_{1}=1.337, a_{2}=0.44, b_{2}=0.92$

that are very close to those reported in [16].

The analysis has been completed with the computation of the statistics of the intermittent events extracted from the $0^{\text {th }}$ mode counterpart. The PDFs are reported in figure 7 a) and (b) whereas the approximating functions and the corresponding uncertainties are given in figure $7(\mathrm{c})$ and $(\mathrm{d})$.

More specifically, with reference to equations (6) and (5), the coefficients obtained from the the $0^{\text {th }}$ statistics are: $a_{1}=0.57, b_{1}=1.3, a_{2}=0.41, b_{2}=0.8$

Even though the coefficients are slightly different, it can be concluded that the model proposed in [16] is valid for both the full pressure signals analyzed therein and their $0^{\text {th }}$ mode counterpart. 
(a)

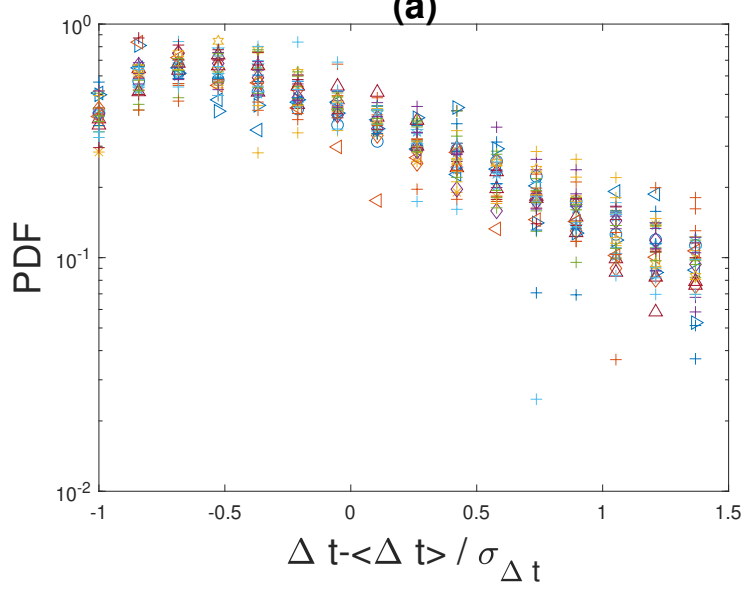

(c)

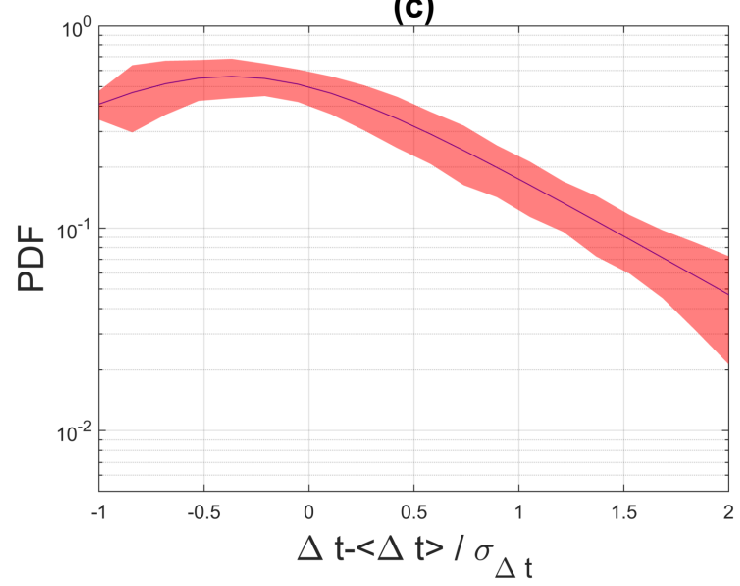

(b)

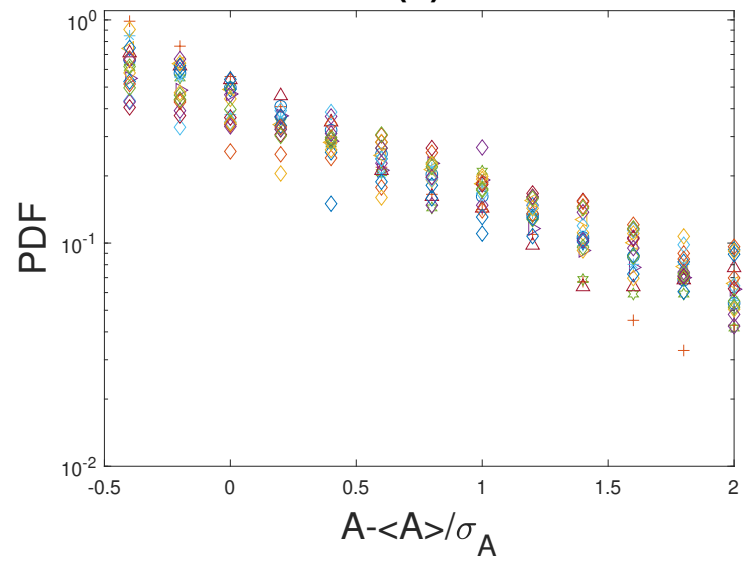

(d)

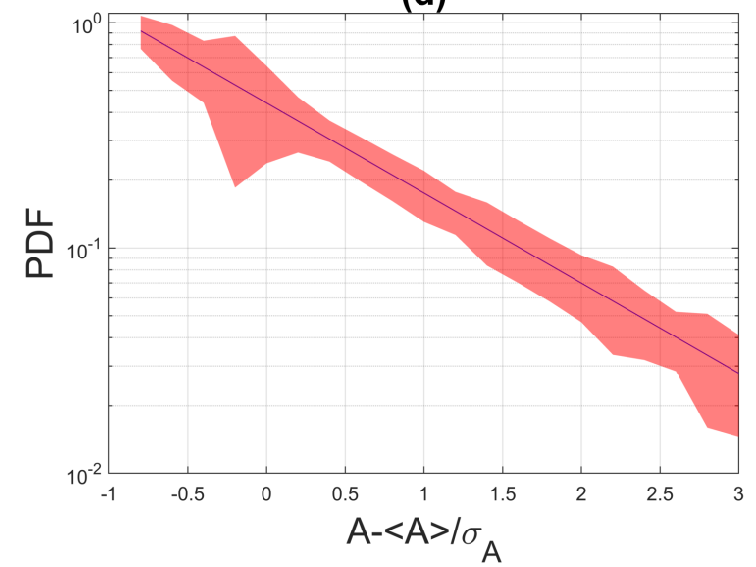

Fig. 6 Probability density functions of the considered signals: (a) $\Delta t$ (b) $A$; (c) and (d) models (5) and (6) with the corresponding uncertainty. 
(a)

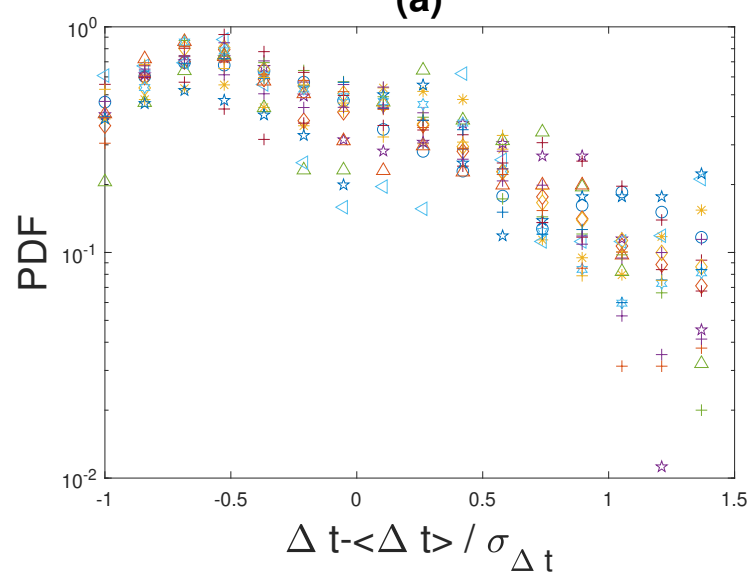

(c)

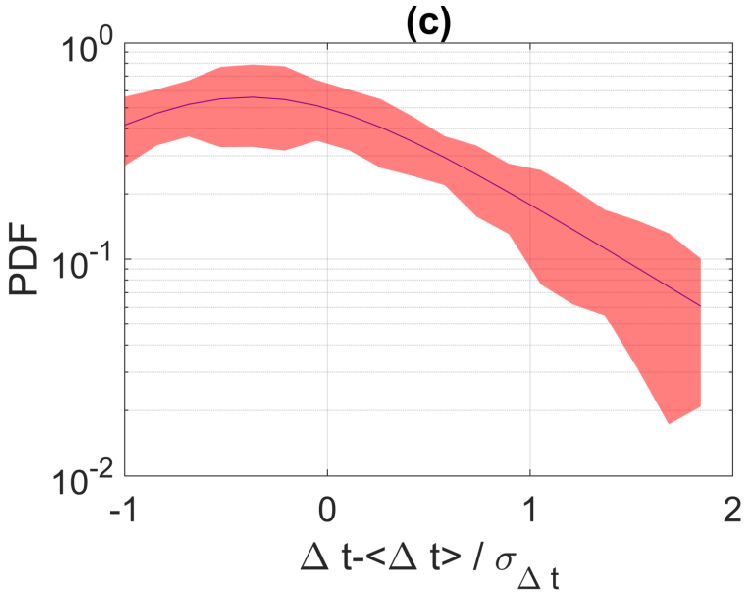

(b)

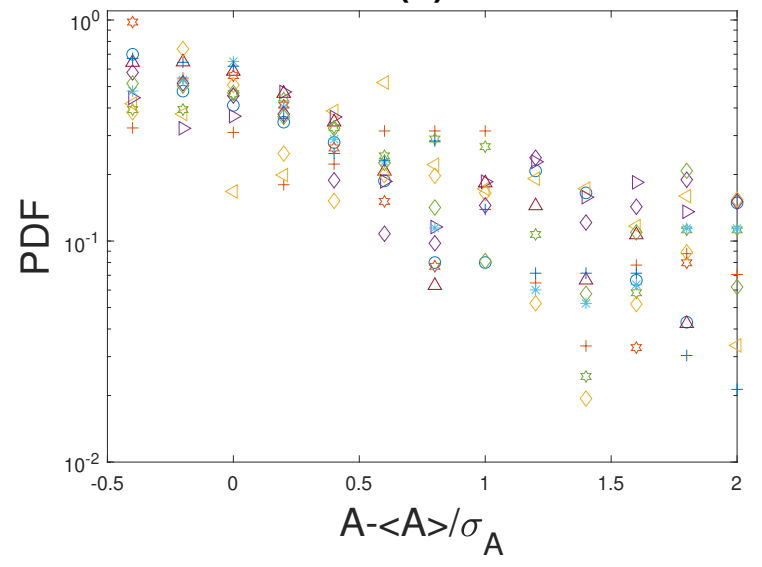

(d)

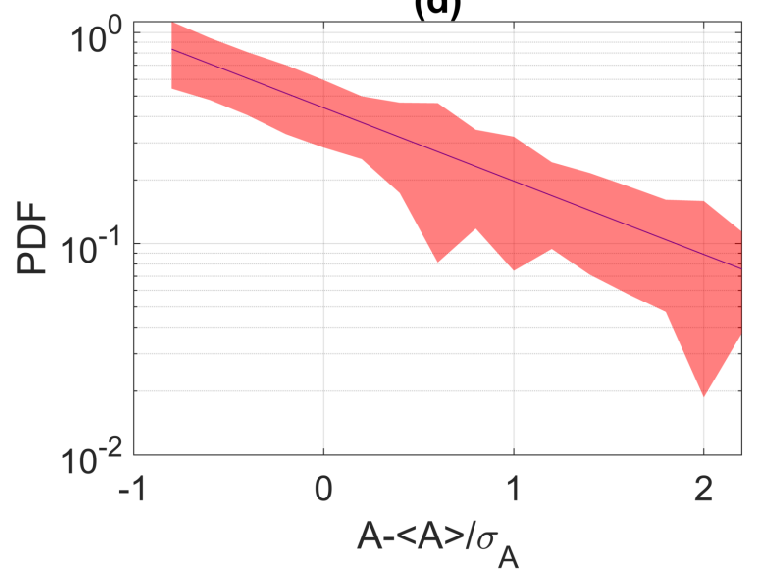

Fig. 7 Probabily density functions of the $0^{t h}$ mode for the considered positions: (a) $\Delta_{t}$ (b) $A$ (c) and (d) models (5) and (6) with the respectively uncertainty. 


\section{Conclusions}

The pressure fluctuations in the near-field of two compressible jets at $\mathrm{M}=0.9$ have been characterized in terms of their intermittent degree with the scope of highlighting the effects of the Reynolds number and the position in the flow. The data used are obtained numerically in a region spanning 7 diameters in the axial and 3 diameters in the radial directions. The pressure data are decomposed azimuthally, and the statistical properties of the 0 -mode component are analyzed as well.

A tracking algorithm based on the computation of the wavelet quantity LIM, has been applied to extract the most energetic events at the frequency of the hydrodynamic pressure bump. By averaging the whole set of available data and following the procedure adopted in [16], a Stochastic model is determined by considering the two variables $t$ and $A$ denoting the intermittent time and the intermittent event amplitude, respectively. The model and the procedure adopted in the present analysis is exactly the same presented in [16] and the used functional forms are identical except for slight differences in the magnitude of the multiplicative coefficients. Specifically, the intermittent time statistics are approximated by an hyperbolic secant function whereas the amplitude statistics by a decaying pure exponential function. The results confirm the generalized validity of the model that is weakly dependent upon $R e$ and upon the position in the near field of the jet. These predictions are shown to be valid also for the statistics extracted from the axisymmetric mode 0 that, in agreement with [17], surprisingly possesses a relevant degree of intermittency.

\section{References}

[1] Camussi, R., Di Marco, A., and Castelain, T., "Statistical analysis of the hydrodynamic pressure in the near field of compressible jets," International Journal of Heat and Fluid Flow, Vol. 64, 2017, pp. 1-9.

[2] Lighthill, M. J., "On sound generated aerodynamically I. General theory," Proceedings of the Royal Society of London. Series A. Mathematical and Physical Sciences, Vol. 211, No. 1107, 1952, pp. 564-587.

[3] Arndt, R. E., Long, D., and Glauser, M., "The proper orthogonal decomposition of pressure fluctuations surrounding a turbulent jet," 1997.

[4] Juvé, D., Sunyach, M., and Comte-Bellot, G., "Intermittency of the noise emission in subsonic cold jets," Journal of Sound and Vibration, Vol. 71, No. 3, 1980, pp. 319-332.

[5] Guj, G., Carley, M., Camussi, R., and Ragni, A., "Acoustic identification of coherent structures in a turbulent jet," Journal of Sound and Vibration, Vol. 259, No. 5, 2003, pp. 1037-1065.

[6] Bogey, C., and Bailly, C., "An analysis of the correlations between the turbulent flow and the sound pressure fields of subsonic jets," Journal of Fluid Mechanics, Vol. 583, 2007, pp. 71-97.

[7] Hileman, J. I., Thurow, B. S., Caraballo, E. J., and Samimy, M., "Large-scale structure evolution and sound emission in high-speed jets: real-time visualization with simultaneous acoustic measurements," Journal of Fluid Mechanics, Vol. 544, 2005, p. 277.

[8] Suponitsky, V., D SANDHAM, N., and Morfey, C. L., "Linear and nonlinear mechanisms of sound radiation by instability waves in subsonic jets," Journal of Fluid Mechanics, Vol. 658, 2010, p. 509.

[9] Kastner, J., Samimy, M., Hileman, J., and Freund, J., "Comparison of noise mechanisms in high and low Reynolds number high-speed jets,” AIAA journal, Vol. 44, No. 10, 2006, pp. 2251-2258.

[10] Bogey, C., Bailly, C., and Juvé, D., "Noise investigation of a high subsonic, moderate Reynolds number jet using a compressible large eddy simulation," Theoretical and Computational Fluid Dynamics, Vol. 16, No. 4, 2003, pp. 273-297.

[11] Cavalieri, A. V., Rodríguez, D., Jordan, P., Colonius, T., and Gervais, Y., "Wavepackets in the velocity field of turbulent jets," Journal of fluid mechanics, Vol. 730, 2013, pp. 559-592.

[12] Zhang, M., Jordan, P., Lehnasch, G., Cavalieri, A. V., and Agarwal, A., “Just enough jitter for jet noise?" 20th AIAA/CEAS Aeroacoustics Conference, 2014, p. 3061.

[13] Tissot, G., Zhang, M., Lajús, F. C., Cavalieri, A. V., and Jordan, P., "Sensitivity of wavepackets in jets to nonlinear effects: the role of the critical layer," Journal of Fluid Mechanics, Vol. 811, 2017, pp. 95-137.

[14] Kearney-Fischer, M., Sinha, A., and Samimy, M., "Intermittent nature of subsonic jet noise," AIAA journal, Vol. 51, No. 5, 2013, pp. 1142-1155. 
[15] Kearney-Fischer, M., "A model function for jet noise events at aft angles and what it says about the statistical relationships of the events," Journal of Sound and Vibration, Vol. 338, 2015, pp. 217-236.

[16] Camussi, R., Mancinelli, M., and Di Marco, A., "Intermittency and stochastic modeling of hydrodynamic pressure fluctuations in the near field of compressible jets," International Journal of Heat and Fluid Flow, Vol. 68, 2017, pp. 180-188.

[17] Camussi, R., and Bogey, C., "Intermittent statistics of the 0-mode pressure fluctuations in the near field of Mach 0.9 circular jets at low and high Reynolds numbers," Theoretical and Computational Fluid Dynamics, Vol. 35, No. 2, 2021, pp. $229-247$.

[18] Bogey, C., "Two-dimensional features of correlations in the flow and near pressure fields of Mach number 0.9 jets," AIAA Scitech 2019 Forum, 2019, p. 0806.

[19] Bogey, C., "Grid sensitivity of flow field and noise of high-Reynolds-number jets computed by large-eddy simulation," Int. J. Aeroacoust., Vol. 17, No. 4-5, 2018, pp. 399-424. https://doi.org/10.1177/1475472X18778287.

[20] Bogey, C., "Acoustic tones in the near-nozzle region of jets: characteristics and variations between Mach numbers 0.5 and 2," $J$. Fluid Mech., 2021. https://doi.org/10.1017/jfm.2021.426

[21] Fuchs, H. V., and Michel, U., "Experimental evidence of turbulent source coherence affecting jet noise," AIAA journal, Vol. 16, No. 9, 1978, pp. 871-872.

[22] Juve, D., Sunyach, M., and Comte-Bellot, G., "Filtered azimuthal correlations in the acoustic far field of a subsonic jet," AIAA Journal, Vol. 17, No. 1, 1979, pp. 112-113.

[23] Meloni, S., Lawrence, J. L., Proenca, A. R., Self, R. H., and Camussi, R., "Wall pressure fluctuations induced by a single stream jet over a semi-finite plate," International Journal of Aeroacoustics, Vol. 0, No. 0, 2020, p. $1475472 X 20930650$. https://doi.org/10.1177/1475472X20930650 URL https://doi.org/10.1177/1475472X20930650

[24] Guj, G., and Camussi, R., "Statistical analysis of local turbulent energy fluctuations," Journal of Fluid Mechanics, Vol. 382, 1999, p. 1. 